\title{
0718 CONFLICTING TRENDS IN FALL INJURY RATES: IMPLICATIONS FOR INJURY PREVENTION
}

W L Watson*, R Mitchell Correspondence: NSW Injury Risk Management Research Centre, BIdg G2, Western Campus, University of New South Wales, Sydney, NSW 2052, Australia

\subsection{6/ip.2010.029215.718}

Background Despite considerable advances in falls prevention research and practice, the rate of falls-related hospitalisations continues to increase. Yet, there is some evidence that hip fracture rates, a major cause of fall-related morbidity, are declining. An examination of trends in types of injuries that contribute to the overall fall injury rate is required to understand these conflicting trends.

Aim To examine the trends in fall-related hospital admissions by injury type in New South Wales (NSW), Australia.

Method A retrospective review of fall-related injury hospitalisations in NSW among individuals aged $65+$ years, by injury type, was conducted from 1 July 1998 to 30 June 2009. Incident cases were identified using the referral source field and direct age standardised admission rates were calculated. Negative binomial regression was used to examine the statistical significance of changes in the trend over time of hospitalised fallrelated injuries by injury type.

Results The overall fall-related hospitalisation rate increased by $1.7 \%$ each year ( $p<0.0001 ; 95 \%$ CI 1.3 to 2.1$)$. However, the fracture rate declined by $-0.4 \%(p<0.03 ; 95 \% \mathrm{CI}-0.8$ to 0$)$ and the rate of non-fractures increased by $6.1 \%$ ( $p<0.0001 ; 95 \% \mathrm{CI}$ 5.5 to 6.7$)$ each year. In particular, severe head injuries, rib(s) and pelvic fracture rates are increasing while hip and forearm fracture rates are declining.

Conclusion It appears that, while falls prevention efforts in NSW are not yet affecting hospitalised falls injury rates, efforts in relation to bone health may be having an effect in preventing fractures. It is likely that the increase in severe head injuries is due to improvements in diagnosis. 